\title{
Reflektieren in Forschungsgemeinschaften: Ansatzpunkte, Formate und Erfahrungen
}

\author{
Petra Wihofszky, Michael T. Wright, Susanne Kümpers, Sandra \\ Layh, Gesine Bär und Ina Schaefer ${ }^{1}$
}

\section{Zusammenfassung}

In der partizipativen Forschung kommt der Reflexion eine wichtige Bedeutung zu. Gegenstand reflexiver Prozesse sind häufig die Frage nach der Entscheidungsmacht im partizipativen Prozess sowie die Phasen und verschiedenen Schritte des Forschungsprozesses. In diesem Beitrag gehen wir der Frage nach, wie in partizipativen Forschungsprojekten reflektiert wird, wie Forschungsgemeinschaften ihre Erkenntnisse nutzen und sie in den Forschungsprozess integrieren. Dazu nähern wir uns zunächst den Begriffen und dem Verständnis von Reflexion und Reflexivität im Kontext der partizipativen Forschung an. Anschließend stellen wir Ansatzpunkte, Formate und Erfahrungen des Reflektierens in Forschungsgemeinschaften vor. Der Beitrag entwickelte sich aus unserer gemeinsamen Arbeit im Forschungsverbund PartKommPlus. Wir

1 Petra Wihofszky | Hochschule Esslingen | petra.wihofszky@hs-esslingen.de

Michael T. Wright | Katholische Hochschule für Sozialwesen Berlin | michael.wright@ khsb-berlin.de

Susanne Kümpers | Hochschule Fulda | susanne.kuempers@pg.hs-fulda.de Sandra Layh | Hochschule Esslingen | sandra.layh@hs-esslingen.de

Gesine Bär | Alice Salomon Hochschule Berlin | baer@ash-berlin.eu

Ina Schaefer | Alice Salomon Hochschule Berlin | ina.schaefer@ash-berlin.eu

(C) Der/die Autor(en) 2020

S. Hartung et al. (Hrsg.), Partizipative Forschung, https://doi.org/10.1007/978-3-658-30361-7_3 
beabsichtigen damit, Reflexion und Reflexivität als methodologische Grundlage partizipativer Forschung weiterzuentwickeln, zu begründen und ihren Mehrwert als eine zusätzliche Erkenntnisquelle sichtbar zu machen.

\section{Schlüsselbegriffe}

Reflexion; Reflexivität; partizipative Forschung; Forschungsgemeinschaft; Supervision

\section{$1 \quad$ Einführung}

Partizipativ zu forschen bedeutet prozessorientiert vorzugehen. Methodologisch liegt der partizipativen Forschung ein zyklisches Modell zugrunde, das zwischen Datensammeln, Handeln und Reflektieren pendelt (Ledwith \& Springett 2010; Moser 1995). Dieses Vorgehen orientiert sich am Aktions-Reflexions-Modell, das sich aus der Aktionsforschung entwickelte (Altrichter 2008; Jacobs 2008). Aktion und Reflexion sind sich wechselseitig ergänzende Bestandteile des partizipativen Forschungsprozesses und werden in der Literatur auch als die Phasen look - think - act beschrieben (Koch et al. 2005). Aus Informationen und Daten werden praxisrelevante Annahmen und Handlungsstrategien generiert, die zur Initiierung von Maßnahmen und Veränderungen führen. Deren Erkenntnisse fließen wiederum in den Forschungsprozess zurück. Reflexion trägt damit entscheidend zum Gelingen partizipativer Forschung bei (Apgar et al. 2017; Borg et al. 2012; Chiu 2006; Dorozenko et al. 2016).

Anhand eines Fallbeispiels aus einem Stadtteilprojekt wollen wir nachfolgend verdeutlichen, wie sich das Reflektieren in einer Forschungsgemeinschaft auf den Projektverlauf auswirken kann. Eine Beschreibung des Stadtteilprojekts findet sich in Wihofszky (2013). Eine Forschungsgemeinschaft meint den Zusammenschluss verschiedener Interessengruppen, wie beispielsweise Bürger*innen, Vertreter*innen der Zivilgesellschaft, Fachkräfte aus dem Gesundheits-, Sozial- oder Bildungswesen, Wissenschaftler*innen und anderen, die zusammenkommen, um einen Forschungsprozess gemeinsam zu gestalten und zu steuern (Wright 2013). Dort werden also verschiedene Wissensformen zusammengeführt, um daraus neues Wissen $\mathrm{zu}$ generieren. Das Stadtteilprojekt verfolgte das Ziel, eine sozialraumorientierte Strategie zur Gesundheitsförderung einzuführen. Die Herausforderung einer sozialraumorientierten Strategie besteht darin, die Fachkräfte und die Bewohnerschaft 
mit ihren unterschiedlichen Rollen, Anliegen und Aufgaben zusammenzubringen. Ein partizipativer Ansatz schien im Fall des Projekts geeignet zu sein, aber nach einem Jahr Präsenz im Stadtteil zeigte sich, dass der Anspruch, partizipativ im Sinne einer geteilten Entscheidungsmacht vorzugehen, nur teilweise eingelöst werden konnte. Den Anstoß für einen Reflexionsprozess gab ein Workshop des Netzwerks „Partizipative Gesundheitsforschung“ (siehe Beitrag 1 Partizipative Forschung - ein Forschungsansatz für Gesundheit und seine Methoden in diesem Buch). Durch das Reflektieren erkannte die Forschungsgemeinschaft des Projekts verpasste Partizipationschancen und Partizipationshindernisse. Vor diesem Hintergrund setzte sich die Forschungsgemeinschaft mit der Frage auseinander, wie zukünftig mehr Partizipation ermöglicht werden könnte. Der im Workshop begonnene Reflexionsprozess wurde im Stadtteil fortgesetzt. Es gelang schließlich, im Prozess nachzusteuern: Zum Beispiel wurde eine Stadtteilbefragung aufsuchend gestaltet, um mit der Bewohnerschaft ins Gespräch zu kommen. Bei der Vernetzung mit Fachkräften wurde unter anderem mit visuell-kreativen Methoden wie dem belebten Soziogramm gearbeitet (Wihofszky 2013). Die Erfahrung zeigt, dass es mittels Reflexion gelingen kann, im Prozess eine neue Richtung einzuschlagen. Dafür ist es notwendig, die eigene Haltung zu überdenken und Zeit und Raum für Kommunikation und Reflexion zu schaffen.

Für die partizipative Forschung unterscheiden Bergold und Thomas (2012) vier Ebenen der Reflexion: die personale Ebene, die Ebene der sozialen Beziehungen zwischen den Beteiligten, strukturelle Bedingungen und die Ebene des Forschungsprozesses. Auf der personalen Ebene führte ein niederländisches Projekt spezielle Trainings für Fachkräfte ein, um eine partizipative Haltung in reflexionsfördernden Prozessen zu vermitteln und einzuüben (Jacobs 2008). Um einen partizipativen Forschungsprozess als Ganzes zu reflektieren, schlagen beispielsweise von Köppen und Kolleginnen (siehe Beitrag 2 Mit vulnerablen Gruppen forschen in diesem Buch) und Engage for Equity (2019) zwei Modelle vor, die unter anderem die Reflexion der sozialen Beziehungen im Projekt vorsehen. Bei solchen Modellen wird jedoch oft nicht erklärt, auf welcher Grundlage und mithilfe welcher Methoden die Beziehungen im Projekt reflektiert werden. Im Forschungsverbund PartKommPlus haben wir uns (siehe Beitrag 1 Partizipative Forschung - ein Forschungsansatz für Gesundheit und seine Methoden in diesem Buch) mit der Frage auseinandergesetzt, wie sich Beziehungen aus dem Forschungsfeld in den Forschungsgemeinschaften reproduzieren und im Geschehen des Verbundes zeigen. PartKommPlus fördert reflexive Praxen, thematisiert Reflektieren als Teil partizipativer Forschung in den Kolloquien und nutzt Reflexion zur Untersuchung von Wirkungen (Impact).

In diesem Beitrag gehen wir zunächst in Abschnitt 2 auf die Begriffe Reflexion und Reflexivität ein. In Abschnitt 3 stellen wir Formen des Reflektierens vor, die 
auf Erfahrungen des Forschungsverbundes PartKommPlus zurückgehen. Diese Formen bilden die Grundlage für ein Modell, das vier Ansatzpunkte und Formate des Reflektierens unterscheidet. Der Schwerpunkt des Beitrags liegt auf Abschnitt 4, in dem wir die vier konzeptionellen Ansatzpunkte konkretisieren und Einblicke in die Praxis des Reflektierens verschiedener Forschungsgemeinschaften geben. Unsere Leitfrage dabei ist, wie Erkenntnisse aus reflexiven Prozessen für die Koproduktion von Wissen im Forschungsprozess genutzt werden können. In Abschnitt 5 ziehen wir ein Fazit und geben einen Ausblick auf die Weiterentwicklung reflexiver Formate und Praxen in der partizipativen Forschung.

\section{Reflexion und Reflexivität}

Reflexion und Reflexivität werden im Fachdiskurs begrifflich differenziert (Tremblay et al. 2013). Fast immer wird dabei auf das von Donald Schön formulierte Konzept der reflective practice zurückgegriffen (Schön 1983; Chiu 2006; Jacobs 2008; Ledwith \& Springett 2010; Tremblay et al. 2013; Wright 2012). Tremblay und Kolleginnen (2013) betrachten reflective practice als eine Form der Reflexion, die in einem professionellen Kontext wie zum Beispiel der Praxis der Gesundheitsförderung angewandt wird. Dabei reicht reflective practice oder reflexive Praxis aus Sicht von Tremblay und Kolleginnen über die Reflexion hinaus, wenn Reflexion, wie Jacobs definiert, nur als ein gedanklicher Prozess des Betrachtens und Bewertens verstanden wird (2008). Reflexive Praxis will Praxis verbessern und verändern (Bisset et al. 2015).

Der Begriff Reflexivität schließt an das Konzept der reflexiven Praxis an. Als Definition des Begriffs Reflexivität schlagen Tremblay und Kolleginnen vor (2013):

"[...] an intentional intellectual activity in which individuals explore or examine a situation, an issue or a particular object on the basis of their past experiences to develop new understandings that will ultimately influence their actions [...]" (S.539).

In der Literatur wird das Verständnis von Reflexivität erörtert und auf Diskurse wie die kritische Theorie und Konzepte wie von Paulo Freire zurückgeführt (Chiu 2006; Ledwith \& Springett 2010). Wir orientieren uns in diesem Beitrag an der vorgestellten Definition von Tremblay und Kolleginnen, die sich deshalb eignet, da mit Reflexivität als eine Form reflexiver Praxis Handlungsorientierung impliziert wird - ein Kernmerkmal von Aktionsforschung und partizipativer Forschung. Das Ziel von Reflexivität besteht darin, Veränderungen im Sinne einer Transfor- 
mation von Lebenswelten anzuregen und zu bewirken (Altrichter 2008; Ledwith \& Springett 2010). Dieses Verständnis geht weit darüber hinaus, was unter Reflexion verstanden wird.

Reflexivität wird in der Literatur häufig in mehrere Ebenen unterteilt. Diese Teilung ist eine analytische, praktisch sind die Ebenen miteinander verwoben und nicht voneinander zu trennen. Eine Zweiteilung nehmen Tremblay und Kolleginnen vor: Sie unterscheiden formative und kritische Reflexivität (2013). Unter formativer Reflexivität verstehen sie die Reflexivität von Professionellen, die Sinnhaftigkeit einer Situation zu erfassen mit dem Ziel, Praxis zu verbessern. Kritische Reflexivität bezieht nach ihrer Auffassung mit ein, Machtverhältnisse zu hinterfragen, moralische und ethische Fragen zu stellen sowie soziale Gerechtigkeit zu thematisieren (Bisset et al. 2015; Tremblay et al. 2013).

Die von uns verwendete Definition von Tremblay et al. (2013) bildet die Basis für eine weitere Unterteilung von Reflexivität in drei Ebenen: technische oder rationalistische Reflexivität, praktische Reflexivität und kritische Reflexivität (Ledwith \& Springett 2010; Wright 2012). Unter technischer oder rationalistischer Reflexivität wird die Überprüfbarkeit von Standards und Normen verstanden, die subjektive, erfahrungsbasierte, intuitive Formen von Wissen ersetzen sollen. Diese Normen werden in Praxisroutinen eingebaut, sodass unabhängig von der Person der Fachkraft die „richtige“ Handlung umgesetzt wird. Diese Reflexion ist eindimensional (single-loop learning, Ledwith \& Springett 2010). Die praktische Reflexivität ergänzt die technische Reflexivität. Bei der praktischen Reflexivität fließt Erfahrungswissen von Fachkräften ein. Verknüpft man praktische Reflexivität und technische Reflexivität miteinander, erreicht man eine zweidimensionale Reflexivität (double-loop learning, Ledwith \& Springett 2010). In der partizipativen Arbeit kommt die kritische Reflexivität als dritte Dimension hinzu. Partizipativ zu arbeiten stellt an Fachkräfte neue professionelle Anforderungen, wie zum Beispiel Routinen zu durchbrechen, flexibel mit Bedürfnissen und Anliegen von Adressat*innen umzugehen, erfordert Kompetenzen wie Dialog- und Kooperationsbereitschaft, die Fähigkeit, einen Konsens zwischen Top-down und Bottom-up zu finden sowie Macht abzugeben und zu teilen. Dasselbe gilt für partizipativ Forschende (Chiu 2006). Unter kritischer Reflexivität wird die Reflexion der eigenen Rolle vor dem Hintergrund gesellschaftlicher Prozesse verstanden, die unter anderem zu Ausgrenzung und Stigmatisierung führen. Diese dritte Dimension kritischer Reflexivität wird damit auch zu einer politischen Dimension (level three learning, Ledwith \& Springett 2010).

Im Rahmen dieses Beitrags, der das Reflektieren in Forschungsgemeinschaften partizipativer Forschungsprojekte beleuchten will, ist insbesondere die Ebene der kritischen Reflexivität von Relevanz. Das Verständnis der kritischen Reflexivität bietet 
Bezugspunkte, wie Beziehungen und Interaktionen in einer Forschungsgemeinschaft untersucht, Machtstrukturen aufgedeckt und benannt werden können. Kritische Reflexivität unterstützt die Beteiligten, sich in einer Forschungsgemeinschaft auf Augenhöhe zu begegnen. Eine kritische Reflexion nimmt soziale Benachteiligung nicht als Merkmal einer Person oder einer Gruppe, sondern als Ausprägung einer gesellschaftlichen Dynamik wahr, zu der Forschende, die in diesen Strukturen arbeiten, (unbewusst) beitragen und die sich in Forschungsgemeinschaften reproduzieren kann. Die kritische Reflexivität fordert auf, sich mit Werten und Machtverhältnissen auseinanderzusetzen (Wright 2012), darauf basierend Praxis $\mathrm{zu}$ verbessern und zu sozialer Weiterentwicklung beizutragen.

\section{$3 \quad$ Ansatzpunkte und Formate des Reflektierens}

Die Mitglieder des Forschungsverbundes PartKommPlus betrachten Reflexion als einen unersetzlichen Teil partizipativer Forschung. Reflexion ermöglicht, die verschiedenen Perspektiven innerhalb einer Forschungsgemeinschaft zu verstehen und macht „echte" Partizipation aller Beteiligten erst möglich (PartKommPlus 2018; Wright et al. 2018b) (zu den Stufen der Partizipation siehe Beitrag 1 Partizipative Forschung - ein Forschungsansatz für Gesundheit und seine Methoden in diesem Buch). Der Forschungsverbund PartKommPlus trifft sich zweimal jährlich zu Kolloquien. In einem der dort stattfindenden Workshops tauschten sich Mitglieder der verschiedenen Teilprojekte und der Koordinierungsstelle zum Thema des Reflektierens aus. Der Austausch gab Einblicke in die konkreten Praxen des Reflektierens.

In allen Forschungsgemeinschaften des Verbundes ist Reflexion ein Bestandteil von Teambesprechungen, Runden Tischen und ähnlichen Treffen einschließlich deren Nachbereitungen. Manche Forschungsgemeinschaften institutionalisierten Reflexion und entwickelten eigens Instrumente oder nutzen Forschungstagebücher, um ihre Erkenntnisse festzuhalten. Andere reflektierten eher informell. Die Workshopteilnehmenden erkannten, dass sich die Formate des Reflektierens und ihre Erfahrungen entlang zweier Achsen zwischen jeweils zwei Polen einordnen lassen: Achse 1 verläuft zwischen den Polen informell und institutionalisiert, Achse 2 zwischen den Polen offen und strukturiert. Kreuzt man die beiden Achsen miteinander, lassen sich vier schematische Felder als Ansatzpunkte definieren, in die die Workshopteilnehmenden ihre Reflexionsformate und Erfahrungen einordnen konnten (siehe Abb. 1): Informell-offenes Reflektieren kann zum Beispiel als kollegialer Austausch verstanden werden, der nicht in einem festgelegten Setting stattfindet; Erkenntnisse werden nicht offiziell protokolliert. Informell-strukturiertes 
Reflektieren formalisiert nicht die Wahl des Settings, aber von allen Treffen und Besprechungen einer Forschungsgemeinschaft werden zum Beispiel Notizen und Memos angefertigt. Institutionalisiert-offenes Reflektieren kann zum Beispiel ein Austausch in Teambesprechungen auf der Grundlage von Forschungstagebüchern sein. Ein Beispiel für institutionalisiert-strukturiertes Reflektieren ist ein Austausch zu festgesetzten Zeitpunkten mithilfe eines Instrumentes wie dem Stufenmodell der Partizipation (siehe Beitrag 1 Partizipative Forschung - ein Forschungsansatz für Gesundheit und seine Methoden in diesem Buch), das einen reflexiven Prozess systematisiert.

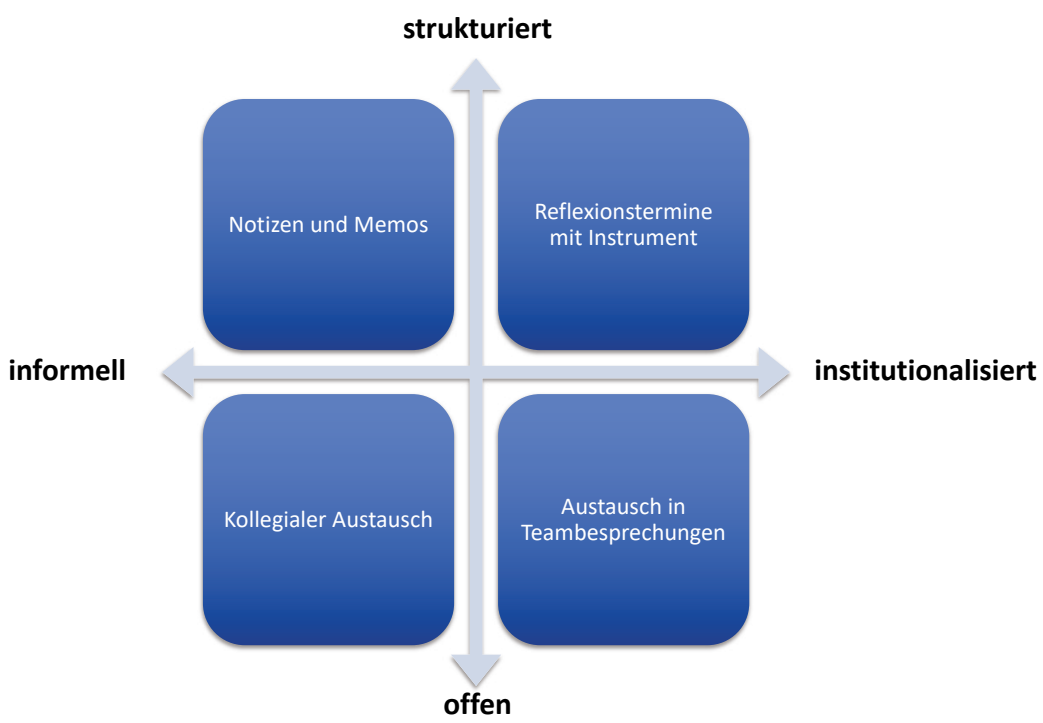

Abb. 1 Schema zu Reflexionsformaten im Forschungsverbund PartKommPlus

Das Schema (Abb. 1) verdeutlicht, in welcher Bandbreite in partizipativen Forschungsprojekten reflektiert werden kann und wie heterogen Formate des Reflektierens sein können. Die Diskussion und der Austausch zwischen den Mitgliedern des Forschungsverbundes PartKommPlus zeigten, dass in allen Forschungsgemeinschaften Facetten aller von uns unterschiedenen vier Ansatzpunkte und Formate des Reflektierens genutzt werden. Unser Schema stellt keine Wertehierarchie der 
Ansatzpunkte dar, sondern dient dazu, sich einzuordnen und gemeinsam über Reflexionsformate zu verständigen.

Als Gegenstände von Reflexion nannten die Workshopteilnehmenden Themen wie zum Beispiel die Zusammenarbeit in der Forschungsgemeinschaft, den Grad an Partizipation, der nach eigener Einschätzung erreicht wird, oder die (Nach-) Wirkungen einer Erhebungssituation in der Rolle als interviewende oder interviewte Person. Anlass, sich in der Forschungsgemeinschaft zusammenzusetzen und zu reflektieren, bot aber auch zum Beispiel das gemeinsame Verfassen eines Artikels, für den die Beteiligten unter anderem Eindrücke verarbeiten und gemeinsam Perspektiven austauschen konnten (siehe Beitrag 4 Photovoice als partizipative Methode: Wirkungen auf individueller, gemeinschaftlicher und gesellschaftlicher Ebene in diesem Buch). Die im Workshop geteilten Erfahrungen machten deutlich, dass je nach Rolle und Hintergrund - der Anspruch an und die Formen von Reflexion differieren. Zum Beispiel unterscheiden sich die wissenschaftlichen Ansprüche und Formate der akademisch Forschenden von den handlungsorientierten Ansprüchen und Formaten anderer Forschungspartner*innen. Nach Aussage einer der Workshopteilnehmenden besteht daher ein Bedarf an Formaten, die für alle Beteiligten verständlich und sinnvoll sind. Diesem Bedarf wird teilweise bereits entsprochen. Teilprojekte des Verbundes entwickelten Formate, die sich speziell an die Praxis und deren Anliegen adressieren (die Teilprojekte und deren Ergebnisse werden auf der Homepage des Forschungsverbundes vorgestellt: http://partkommplus. de). Als eine weitere Herausforderung beschrieben die Workshopteilnehmenden, die Erkenntnisse aus den reflexiven Prozessen wieder in den Forschungsprozess einfließen zu lassen, wie es das Modell von Aktion und Reflexion vorschlägt. An dieser Herausforderung setzt dieser Beitrag an und stellt sich zunächst der Aufgabe, konkrete Formate des Reflektierens im nachfolgenden Abschnitt 4 herauszuarbeiten.

\section{$4 \quad$ Einblicke in die Reflexionspraxis der Forschungsgemeinschaften}

Im Folgenden geben vier Forschungsgemeinschaften des Forschungsverbundes PartKommPlus Einblicke in ihre Praxen des Reflektierens. Die von uns in Abschnitt 3 unterschiedenen Ansatzpunkte - informell-offenes, informell-strukturiertes, institutionalisiert-offenes und institutionalisiert-strukturiertes Reflektieren kommen in jeder der Forschungsgemeinschaften vor, aber es haben sich Schwerpunkte und spezifische Reflexionsformate projekt-, situations- und phasenbedingt herausgebildet. Diese Schwerpunkte greifen wir in der folgenden Darstellung auf 
und beschreiben sie, wohlwissend, dass sie nur einen Teil der jeweiligen reflexiven Praxis der Forschungsgemeinschaften abbilden.

\subsection{Informell-offenes Reflektieren}

Im Folgenden werden Charakteristika und Funktionen von unstrukturierten und ungeplanten Reflexionssituationen betrachtet und anhand von Prozessen konkretisiert, die zwischen der Ebene eines Teilprojekts (hier insbesondere „Age4Health - Gesunde Stadtteile für Ältere“) und des Verbundes stattfanden. Das partizipative Forschungsprojekt „Age4Health - Gesunde Stadtteile für Ältere“ untersucht und fördert in zwei hessischen Kommunen Gestaltungsmöglichkeiten für die gelingende Beteiligung Älterer - insbesondere auch in schwierigen Lebenslagen. Geforscht wird gemeinsam mit Kooperationspartner*innen sowie professionellen und zivilgesellschaftlichen Akteur*innen und Zielgruppen im Stadtteil (Fallstudie Kassel-Bettenhausen) sowie im ländlichen Raum (Fallstudie Witzenhausen). Ziel ist die Entwicklung von inklusiven und gesundheitsförderlichen Quartieren und Nachbarschaften (Kümpers et al. 2017).

Der Forschungsverbund PartKommPlus, zu dem „Age4Health - Gesunde Stadteile für Ältere“ als Teilprojekt gehört, stellte ein bis dahin eher unbekanntes und ungeübtes (partizipatives) Kooperationsformat dar. Mehrere partizipativ vorgehende Teilprojektteams (von akademisch Forschenden und Mitforschenden) und ein koordinierendes Team, das die Verantwortung für den gemeinsamen Erkenntnisprozess hatte, trafen regelmäßig zusammen. Das gemeinsame Ziel des Verbundes und seiner Teilprojekte war es, Partizipation im Rahmen integrierter kommunaler Strategien der Gesundheitsförderung unter unterschiedlichen Bedingungen zu untersuchen. Die Teilprojekte waren aber darüber hinaus, insbesondere in den ersten Jahren, intensiv mit ihren je eigenen (lokalen) Prozessen und Suchbewegungen hinsichtlich ihrer höchst unterschiedlichen konkreten Thematiken beschäftigt. Weder auf der Ebene der Teilprojekte noch auf der Ebene des Forschungsverbundes konnten alle beteiligten akademisch Forschenden und Mitforschenden durchweg auf in vergleichbaren Kontexten erprobte Routinen der Kooperation, der Wissensgenerierung und der Reflexion zurückgreifen. Daher erlebten sie in den Anfangsjahren teilweise Situationen, die von Unsicherheit, offenen Suchprozessen und von empfundener, wenn auch selten offen ausgesprochener Konkurrenz charakterisiert waren. Dabei ging es immer wieder um ein vertieftes (Selbst-)Verständnis und die manchmal mühsame Umsetzung der Prinzipien partizipativer Forschung sowie um die Suche nach gemeinsamen Strategien der Bewältigung. 
Teil des Austauschs innerhalb der Teilprojekteteams waren informelle Reflexionssituationen. Damit sind beispielsweise informelle Gespräche über Projektthemen oder -verläufe gemeint, die oft am Rande strukturierter Arbeitssituationen zufällig entstehen oder (halb-)bewusst herbeigeführt werden. In klassischen wissenschaftlichen Hierarchien und Organisationen mit festgelegten Forschungszielen und -schritten scheinen solche Situationen randständig und unbedeutend. In der partizipativen Forschung hingegen haben informelle (Gesprächs-)Situationen wichtige Funktionen für den Aufbau und die Intensivierung von Beziehungen und Vertrauen - auch über die empfundenen oder tatsächlichen Grenzen zwischen den unterschiedlichen Akteur*innen (den akademisch Forschenden, den Mitforschenden und Praxispartner*innen) hinweg (Borg et al. 2012). Durch diese Situationen wird ein offener und reflexiver Austausch ermöglicht. Hier entstehen wichtige Ausgangspunkte für Reflexivität und die Entwicklung tendenziell hierarchiefreier Diskurssituationen. Noch nicht formulierte Wahrnehmungen, Ideen oder Störungen im Hinblick auf die Projektverläufe werden in solchen informellen Situationen formuliert und ,probebesprochen', dabei erstmalig oder weiter, tiefer und genauer durchdacht. In der Folge werden sie möglicherweise in eine formellere Gesprächssituation eingebracht. Hier entsteht ein Freiraum für alle Projekt- bzw. Verbundteilnehmenden, unabhängig von ihrer Position im Verbund und auch für die im öffentlichen Diskurs weniger Geübten, ihre Anliegen einzubringen. Die Entwicklung vom Impliziten (Wahrnehmung, Unklarheit, Störung, Anliegen etc.) zum Expliziten (Tagesordnungspunkt, neuer Aspekt einer Forschungsfrage, neue Projektinitiative, Publikationsthema etc.) nimmt häufig hier ihren Ausgangspunkt. Typische Prozesslinien verlaufen vom Impliziten zum Expliziten, vom Individuellen zum Kollektiven und (nicht immer) vom Konkreten zum Abstrakten.

Die Bedeutung und Funktion informeller Reflexionssituationen für den Forschungsprozess soll im Folgenden an einem Beispiel nachgezeichnet werden: Wir stellen dar, wie sich ein Forschungsdiskurs aus informeller Reflexion zu der Frage entwickelt, ob und wie die Beteiligung benachteiligter Gruppen im Forschungsprozess gelingt.

Zentrales Prinzip Partizipativer Gesundheitsforschung ist die Verringerung gesundheitlicher Ungleichheit. Damit steht die Arbeit mit gesundheitlich benachteiligten Gruppen im Vordergrund - und verbindet sich mit der in der Forschungsliteratur oft absolut formulierten Forderung, alle Gruppen müssten von Anfang an gleichberechtigt an allen Schritten der Forschung beteiligt sein. In Projekten, die in nicht-institutionellen Settings (beispielsweise Stadtteilen, Quartieren/ Nachbarschaften) arbeiten, müssen aber der Kontakt zu und die Kooperation mit Zielgruppen häufig erst aufgebaut werden. Das ist voraussetzungsvoll und oft nicht sofort und nicht immer auf direktem Wege erreichbar (Wright 2012). In beiden 
Fallstudien des Projekts Age4Health wurde beispielsweise zunächst mit dem Format Runder Tisch gearbeitet. Benachteiligte Ältere im Stadtteil wurden anfangs kaum erreicht. Erst im Projektverlauf und mit Initiativen, die aus dem Runden Tisch entstanden waren, konnten benachteiligte Ältere partizipieren - jedoch häufig mit abgestuften Partizipationsformen. Diese Situation begründete ein Unbehagen, das insbesondere bei den gegenseitigen Präsentationen der Arbeit anlässlich der Treffen des Forschungsverbundes spürbar wurde. Dieses Unbehagen, den Anforderungen an die Prinzipien partizipativer Forschung nicht umfänglich zu genügen, wurde zunächst mehrfach in informellen (und vertrauensvollen) Gesprächssituationen formuliert. Aus dem Unbehagen konnten zunehmend inhaltliche Fragen entwickelt werden: zum einen kritische Fragen an die Literatur zur partizipativen Forschung, die ähnliche Schwierigkeiten selten thematisiert, zum anderen die Frage, wie sich die komplexen Zusammenhänge von Biografie, Kompetenz und Partizipation in konkreten partizipativen Forschungsprozessen verstehen und bearbeiten lassen. $\mathrm{Zu}$ diesen Fragen entstand zunächst eine kleine teilprojektübergreifende Arbeitsgruppe im Verbund. Auf Verbundtreffen wurden zweimal Workshops zum Thema veranstaltet und Daten aus den verschiedenen Teilprojekten gesammelt; ein Artikel dazu ist geplant.

\subsection{Informell-strukturiertes Reflektieren}

Im Folgenden wird als ein Beispiel für informell-strukturiertes Reflektieren der Ansatz des Reflektierens im Teilprojekt „Kommunale Entwicklung von Gesundheitsstrategien: Wissenschaft und Praxis im Dialog“ (KEG) beschrieben. Informell-strukturiertes Reflektieren findet nicht in einem festgelegten Setting statt, aber alle Treffen und Besprechungen einer Forschungsgemeinschaft können als Gegenstand des Reflektierens genutzt werden. KEG setzte sich in zwei Fallstudien in Hamburg und Esslingen das Ziel, Kenntnisse über gesundheitsbezogene Anliegen in Stadtteilen zu gewinnen und gesundheitsfördernde Strategien auf kommunaler Ebene voranzubringen. Dem Projekt liegt die Annahme zugrunde, dass der Auf- und Ausbau von kommunalen Gesundheitsstrategien von einer gelingenden Kommunikation zwischen beteiligten Institutionen und Akteur ${ }^{\star}$ innen vor Ort abhängt. Im Mittelpunkt des Erkenntnisinteresses standen deshalb nicht nur die gesundheitlichen Anliegen im Stadtteil, sondern auch die kommunikativen Prozesse, die sich in den Forschungsgemeinschaften an der Schnittstelle zwischen Forschung, Praxis und kommunalem Handeln entfalten.

$\mathrm{Zu}$ der Forschungsgemeinschaft der Hamburger Fallstudie gehörten auf Landesebene Fachkräfte der Landesvereinigung für Gesundheitsförderung und der 
Behörde für Gesundheit und Verbraucherschutz. Unter den beteiligten Fachkräften auf Bezirksebene war unter anderem die Koordinatorin eines Netzwerks auf Stadtteilebene. Wissenschaftlerinnen der Hochschule wirkten ebenfalls mit. Die Forschungsgemeinschaft bildete den Dreh- und Angelpunkt und traf sich in regelmäßigen Abständen - in ihr wurden gemeinsam Entscheidungen getroffen und Forschungsschritte geplant und umgesetzt (Cook et al. 2018).

Jedes Treffen in der Forschungsgemeinschaft, wie zum Beispiel Teambesprechungen, Stadtteilveranstaltungen, Kolloquien des Verbundes, wurde zu einem möglichen Reflexionsraum und Lernort. Informelles Reflektieren bedeutete bei KEG, dass ein Termin nicht dafür vereinbart werden musste, sondern Reflektieren als Teil des gemeinsamen Prozesses verstanden wurde. Nur in zwei besonderen Situationen während des Forschungsprozesses wurden zusätzlich spezielle Reflexionstermine notwendig. Einer der beiden wurde anberaumt, da sich keine weiteren Fachkräfte aus dem Stadtteil fanden, die an KEG als Mitforschende mitwirken wollten und/oder konnten. Es bestand daher Bedarf an gemeinsamer Reflexion und Lösungssuche in der bestehenden Forschungsgemeinschaft. In einem zweiten Reflexionstermin wurde die Zusammenarbeit in der Begleitgruppe retrospektiv aufgearbeitet und Strategien für die künftige gemeinsame Arbeit im Forschungsprojekt entwickelt. In diesem zweiten Termin wurde systematischer als im ersten vorgegangen: Die Beteiligten reflektierten entlang von Fragen und adaptierten die Methode Story Dialogue (Labonte et al. 1999).

Alle diese sowie weitere Treffen im Projekt KEG wurden durch die Wissenschaftlerinnen der Hochschule als strukturierendes Verfahren protokolliert. Die so entstandenen Protokolle wurden in der Forschungsgemeinschaft gemeinsam abgestimmt und in einer internen Datenbank als Prozessdokumentation systematisch geordnet abgelegt. Ziel der Prozessdokumentation war es, die kommunikativen Prozesse in der Forschungsgemeinschaft zu erfassen, sie als Daten zu sammeln, zu systematisieren und einer gemeinsamen Auswertung zugänglich zu machen. Mit der Prozessdokumentation sollten reflexive Daten zusammengeführt werden, die auch zur Beantwortung von Forschungsfragen genutzt werden können. In der Tat erwies sich die Prozessdokumentation in der Auswertung des partizipativen Forschungsprozesses als hilfreich. Auf der Grundlage der Prozessdokumentation war es möglich, die Etappen des Forschungsprozesses gemeinsam zu rekonstruieren, durchzusprechen, Perspektiven auf diesen auszutauschen und zu diskutieren und daraus einen Artikel zu verfassen. Die Prozessdokumentation diente auch als Datenpool für die Wirkungsanalysen, die von den Teilprojekten im Forschungsverbund PartKommPlus erstellt werden. Das beschriebene Vorgehen wurde im Projektverlauf der zweiten Förderphase wegen des hohen Arbeitsaufwandes jedoch angepasst. Das Protokollieren erfolgte dann nicht mehr prozessorientiert, sondern ergebnisorien- 
tiert. Archiviert wurden nur noch die in der Forschungsgemeinschaft genehmigten Protokolle, da vertrauliche Schriftstücke, wie zum Beispiel Gedächtnisprotokolle, sensible Daten sowie Perspektiven enthalten, die unter Umständen nicht von allen Beteiligten der Forschungsgemeinschaft geteilt werden. Diese Dokumente ebenso wie die Protokolle gemeinsam zu reflektieren und die Schriftform abzustimmen, hätte die zeitlichen Ressourcen des Projekts zu stark beansprucht. Außerdem zeigte sich, dass das Protokollieren reflexiver Prozesse von den Beteiligten in der jeweiligen Situation des Reflektierens als hemmend erlebt werden kann: Alles, was geäußert wird, wird zu Daten und kann im Forschungsprozess verwendet werden.

Der Vorteil eines strukturierten Erfassens reflexiver Prozesse liegt darin, dass kommunikative Daten, die sonst implizit bleiben, gesammelt und für die Auswertung und Bewertung eines Projekts zugänglich werden. Der Nachteil zeigte sich für uns in einem hohen Arbeitsaufwand. Wir stellten auch fest, dass das strukturierte Reflektieren, das sich bei KEG darin zeigte, alle Geschehnisse in Protokollen festzuhalten, sich auch auf die Prozesseigner*innenschaft eines Projekts auswirken kann. Das strukturierte Vorgehen festigt nach unseren Erfahrungen die Deutungsmacht derjenigen, die Protokolle verfassen, selbst wenn diese Protokolle, wie in KEG, mit allen Beteiligten abgestimmt werden. Erst als wir die Form des Protokollierens und Dokumentierens änderten, stärkte sich aus der Perspektive der beteiligten Wissenschaftler*innen die Prozesseigner*innenschaft aller Partner*innen der Forschungsgemeinschaft und förderte eine gleichberechtigte Zusammenarbeit.

\subsection{Institutionalisiert-offenes Reflektieren}

Institutionalisiert-offenes Reflektieren, das zum Beispiel in Form eines kollegialen Austauschs in Teambesprechungen stattfinden kann, beschreiben wir am Beispiel der Koordinierungsstelle des Forschungsverbundes PartKommPlus. Der Verbund besteht aus acht Verbundpartner*innen (sieben Teilprojekten und einem weiteren Partner, der die Kommunikationsstrategie des Verbundes stärkt) und wird von einer Koordinierungsstelle an der Katholischen Hochschule für Sozialwesen Berlin geleitet. Auch auf der Ebene der Koordination nimmt die Reflexion eine zentrale Rolle ein, um die sozialen Beziehungen im Rahmen eines partizipativen Prozesses besser verstehen und moderieren zu können. Hier handelt es sich in erster Linie um die Beziehungen zwischen der Koordinierungsstelle und den Verbundpartner*innen, aber auch um die Beziehungen der Verbundpartner*innen untereinander.

Die Koordinierungsstelle nutzt verschiedene Formen der Reflexion, auch die institutionalisiert-offene Form der wöchentlichen Reflexionen unter den Mitarbeitenden im Rahmen des Teamgesprächs. Zwei Tagesordnungspunkte verankern 
diese Reflexionen. Der eine („Beiträge aus den Tagebüchern: Lessons Learned“) befasst sich mit den neuen Erkenntnissen der Mitarbeitenden aufgrund ihrer Erfahrungen in der vorausgegangenen Woche. Die Wortmeldungen der Teammitglieder basieren oft auf Einträgen aus ihren Forschungstagebüchern. Der zweite Tagesordnungspunkt („Orange Box: Impact-Meldungen“) beschäftigt sich mit den Eindrücken der Mitarbeitenden in Bezug auf die Auswirkungen der Arbeit der Koordinierungsstelle, sowohl innerhalb als auch außerhalb des Verbundes. Der Begriff „Orange Box“ ist der Praxis einer australischen Kollegin entlehnt, die in ihren partizipativen Forschungsprojekten eine orangenfarbige Kiste aufstellt, in die alle Beteiligten fortlaufend Kärtchen einstecken können, auf denen beobachtete Auswirkungen des Projekts notiert sind. Dahinter steht die Überlegung, dass viele Auswirkungen der Projektarbeit nicht erfasst werden, wenn erst zum Schluss evaluiert wird. Die Kiste ermöglicht das kontinuierliche Einsammeln von Eindrücken, die später ausgewertet werden können. Das Team in der Koordinierungsstelle sammelt seine Eindrücke in einer virtuellen Orange Box, auch in Bezug auf die Beziehungen zwischen der Koordinierungsstelle und den Verbundpartner*innen und den Verbundpartner ${ }^{\star}$ innen untereinander.

Die beiden Tagesordnungspunkte stellen eine institutionalisierte Form der Reflexion dar: Reflexion findet als fester Bestandteil der Geschäftsordnung statt und wird protokolliert. Der Austausch selbst ist aber offen, die Mitarbeitenden tauschen sich ohne vorgegebene Struktur über ihre Erkenntnisse und Eindrücke aus. Durch diese offene, dialogische Form regen die Beteiligten sich gegenseitig zu einer tieferen und auch breiteren Reflexion an. So ergibt sich eine facettenreichere und auch differenziertere Einschätzung als durch eine bloße Zusammenstellung der Meldungen der einzelnen Teammitglieder.

Im Rahmen der Aufgabe der Koordinierungsstelle, die Auswirkungen des Verbundes zu erarbeiten, werden die Aussagen aus den Teamgesprächen zusammengetragen und inhaltsanalytisch ausgewertet, um in Kombination mit anderen Datenquellen als Grundlage für die Wirkungsbeschreibung des Verbundes, die sogenannten Impact-Narrative, zu dienen.

\subsection{Institutionalisiert-strukturiertes Reflektieren}

Unter institutionalisiert-strukturiertem Reflektieren verstehen wir einen Austausch $\mathrm{zu}$ festgesetzten Zeitpunkten mithilfe eines Instrumentes, wie im Folgenden am Beispiel des Teilprojekts „ElfE - Eltern fragen Eltern“ dargestellt wird. Thema des Teilprojekts „ElfE - Eltern fragen Eltern“ ist die Stärkung gesundheitlicher Chancengleichheit in der frühen Kindheit. Hier wird vor allem die Kindertagesstätte 
(Kita) als zentraler Interventionsort gesehen. Nachweislich leistet der Kita-Besuch einen positiven Beitrag zum gesunden Aufwachsen, doch profitieren nicht alle Kinder gleichermaßen davon (Robert Koch-Institut 2008; Alice Salomon Hochschule (ASH) \& Gesundheit Berlin Brandenburg (GBB) 2016). Dieser Thematik wurde gemeinsam mit Eltern in einem partizipativen Peer-Forschungsprozess nachgegangen. Gemeinsam legte die Forschungsgemeinschaft fest, auf die bislang zu wenig beachtete Schnittstelle der Zusammenarbeit zwischen Eltern und Kita-Fachkräften zu fokussieren. Angesiedelt war der Prozess in zwei Fallstudien-Kommunen, einer großstädtischen und einer ländlichen. Über je eine Steuerungsgruppe war der Forschungsprozess mit den kommunalen Strukturen verbunden. ${ }^{2}$

Der Forschungsprozess verlief in der ersten Förderphase über anderthalb Jahre. Es waren drei Forschungsteams beteiligt, die jeweils aus Eltern als Peerforschende, akademisch Forschenden sowie Praxispartner*innen (zum Beispiel Familienzentrum) bestanden (Schaefer et al. 2019). Die Forschungsgruppen und die Steuerungsgruppen nutzten für ihre Reflexionsprozesse unter anderem das für sie entwickelte strukturierte Reflexionsinstrument „Stufenleiterabfrage“. Damit sollte prozessbegleitend die partizipative Gültigkeit des Projekts überprüft werden (Wright 2013). Impulsgebend für die Entwicklung des Instruments war das PaKoMi-Projekt, in dem ex post die Beteiligung der verschiedenen Partner ${ }^{\star}$ innen anhand der Stufenleiter der Partizipation (siehe Beitrag 1 Partizipative Forschungein Forschungsansatz für Gesundheit und seine Methoden in diesem Buch) bewertet wurde (von Unger 2012). Ein prozessbegleitendes Instrument zur Reflexion der partizipativen Gültigkeit lag bislang nicht vor.

In einem ersten Schritt wurde zunächst die Stufenleiter entsprechend der ElfE-Projektlogik verändert und die Stufen 7 und 8 zu ,geteilte Entscheidungsmacht“ zusammengezogen (siehe Abb. 2). Jede Stufe wurde mit einem erklärenden Satz operationalisiert und von den anderen Stufen abgegrenzt. So heißt es beispielsweise für „Einbeziehung“: „Ich berate die anderen Beteiligten in wichtigen Fragen, überlasse ihnen aber die Entscheidung." Im Vergleich dazu lautet die Erklärung bei „geteilter Entscheidungsmacht“: „Ich treffe sämtliche Entscheidungen mit.“ Die Erarbeitung verständlicher Formulierungen war ein langer Prozess. Durch die akademisch Forschenden erarbeitete Vorschläge wurden jeweils mit allen Beteiligten in den Forschungsgruppen erprobt, d.h. angewendet und dann reflektiert. Anfangs wurden noch alle acht Stufen abgefragt. Allerdings lag das Spektrum der Antworten jeweils im Bereich der Vor- und der Partizipationsstufen, sodass eine

2 Zur Zusammensetzung der Steuerungsrunde siehe http://partkommplus.de/teilprojekte/ elfe2/elfe-erste-foerderphase/ [Zugriff: 4. Oktober 2019]. 
Reduktion um die Stufen „Selbstorganisation (Stufe 9), Anweisung (Stufe 2) und Instrumentalisierung (Stufe 1) zur besseren Handhabbarkeit angemessen schien.

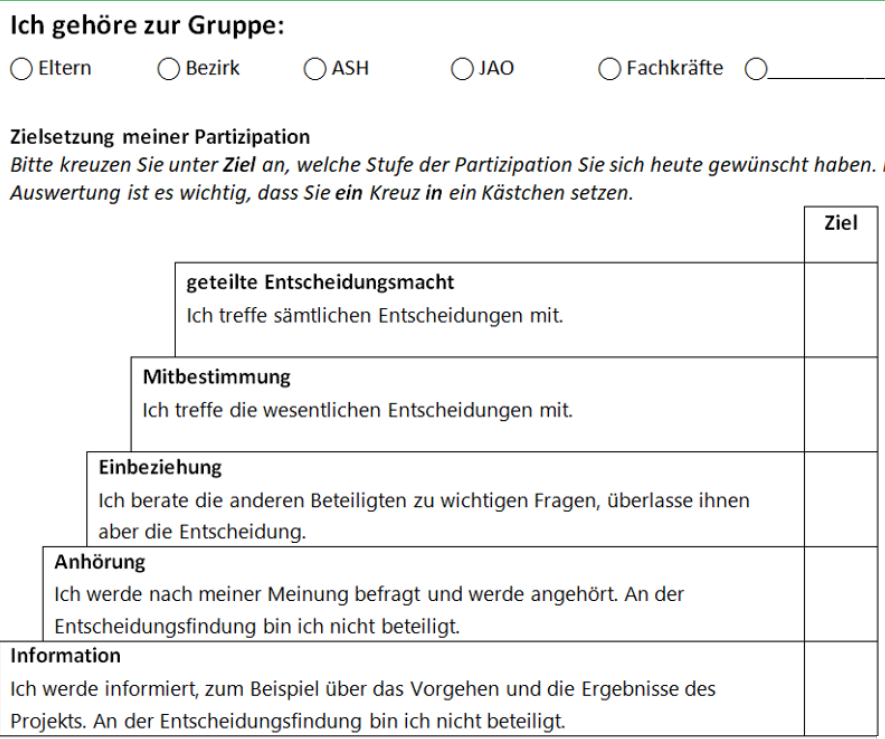

Abb. 2 Ausschnitt aus dem Fragebogen „Messung der Partizipation in ElfE/ElfE ${ }^{2 “}$

Neben der Abfrage der abgebildeten Zielsetzung der Partizipation wurde in dem Fragebogen auf die gleiche Weise die Ist-Situation, d.h. die realisierte Partizipationsmöglichkeit, erfragt. Außerdem wurde unter anderem erhoben, ob ausreichend Gelegenheit zur Information, als Voraussetzung für eine Entscheidungsteilhabe, bestand. Ein Informationsblatt zur Einführung des Fragebogens wurde außerdem eingesetzt.

In allen Forschungswerkstätten bzw. Workshops sowie in allen Sitzungen der Steuerungsgruppe wurde an alle Teilnehmenden je ein Fragebogen verteilt. Die Bögen wurden nicht namentlich ausgefüllt. In einem Ankreuzfeld erfolgte aber eine Zuordnung (und auch Auswertung) zu den beteiligten Gruppen (siehe Abb. 2). Die Fragebögen wurden zusammenfassend ausgewertet. Die Ergebnisse wurden grafisch aufbereitet und in das jeweils folgende Treffen eingebracht. Im Fokus der Betrachtung standen weniger das erwünschte bzw. erzielte Ausmaß an Partizipation 
insgesamt als vielmehr die Differenzen zwischen den einzelnen Angaben. ${ }^{3}$ Diese wurden als Hinweise darauf gewertet, dass in der Zusammenarbeit Unstimmigkeiten wahrgenommen wurden. Wurden Differenzen festgestellt, reflektierten die Forschungsteams, d.h. Eltern, akademisch Forschende sowie Praxispartner ${ }^{\star}$ innen gemeinsam die Gründe der dissonanten Bewertung und diskutierten, wie die Zusammenarbeit verbessert werden könnte.

Der Fragebogen und die Auswertung lassen sich nach Abschluss des Entwicklungsprozesses ohne großen Aufwand gut routinemäßig einsetzen. Der Einsatz will aber wohl überlegt sein, da die Erfahrungen mit dieser strukturierten Reflexion gemischt sind: Zu den Stärken zählt die Herausbildung einer „Reflexionsroutine“ unter den Beteiligten. Informationsmöglichkeiten, Beteiligungserwartungen und genutzte Beteiligungsspielräume werden kontinuierlich zum Thema gemacht. Das sensibilisiert die Beteiligten auch für die Partizipation der anderen Partner*innen. Für die Prozessmoderation ist das Kennenlernen der Beteiligungswünsche ebenso wertvoll wie das Erkennen erweiterter und enttäuschter Mitwirkungsmöglichkeiten. Die Nachbesprechungen führten immer wieder zu Änderungen im Prozess im Sinne einer reflexiven Praxis. Zugleich zeigte sich, dass der Fragebogen nicht selbsterklärend ist und sein Einsatz eine aktive Begleitung erfordert. Es musste jeweils klargestellt werden, auf welche Periode sich die Abfrage bezieht. Außerdem mussten sich die Teammitglieder darüber verständigen, welche Informationsmöglichkeiten und welche Entscheidungen in dieser Periode zur Wahl gestanden hatten. „Nebenbei“ lässt sich der Fragebogen also nicht einsetzen. Aber wenn er routinemäßig mit ausreichend Zeit angewandt wird, kann er eine wertvolle Basis für eine kritische Prozessreflexion bilden.

\section{$5 \quad$ Fazit und Ausblick}

Die Beschreibungen zeigen, wie sich die von uns unterschiedenen Formate des informell-offenen, informell-strukturierten, institutionalisiert-offenen und institutionalisiert-strukturierten Reflektierens in der Praxis umsetzen lassen. In den Beschreibungen zeigen sich auch Vor- und Nachteile der jeweiligen Ansatzpunkte

3 Auch wenn die Stufenleiter dies suggeriert, geht es in der partizipativen Arbeit nicht zwangsläufig darum, eine möglichst hohe Stufe der Partizipation zu erreichen. Je nach Kontext kann eine niedrigere Stufe durchaus angemessen und erwünscht sein. So wählten einige Mitglieder der Steuerungsrunde beispielsweise die Stufe 5 „Mitentscheidung“ als Ziel. In der Zusammenarbeit mit den Eltern wurde allerdings die „geteilte Entscheidungsmacht" angestrebt. 
und Formate, wie zum Beispiel der hohe Arbeitsaufwand bei einem strukturiert geprägten Vorgehen, die Chancen, Neues zu entdecken, wenn informelles Reflektieren ermöglicht wird, oder die Institutionalisierung reflexiver Praxen, die das Betrachten von Prozessen als festen Bestandteil integriert und ermöglicht, dass sich Routinen bilden können. Unsere Erfahrungen zeigen, dass es sich empfiehlt, sich in einer Forschungsgemeinschaft nicht auf Formate festzulegen, sondern im Prozess offen und flexibel zu bleiben, reflexive Praxen und Instrumente anzupassen und weiterzuentwickeln, sowie Stärken und Herausforderungen von Formaten abzuwägen. In unseren Beispielen wird auch deutlich, dass jedes der Formate helfen kann, neue und ergänzende Erkenntnisse zu generieren. Wir legten exemplarisch dar, dass reflexiv gewonnene Daten zum Beispiel in die Prozess- und/oder Wirkungsbewertung von Forschungsgemeinschaften einfließen können, wie im Fall von PartKommPlus in die Erstellung von Impact-Narrativen.

Als Anregungen zur Weiterentwicklung reflexiver Praxen stellten sich uns bei der Aufarbeitung unserer Vorgehensweisen folgende Fragen:

- Reflektieren bringt Daten durch eine Innenschau hervor und partizipative Forschung ist per se subjektiv geprägt, aber worin könnten Gefahren und Risiken liegen, wenn reflexive Erkenntnisse als Datenquelle genutzt werden?

- Wie können blinde Flecken erkannt werden, die eventuell zu einer einseitigen Interpretation von Daten führen, in der sich Machtunterschiede niederschlagen?

- Wie lassen sich blinde Flecken gerade durch kritische Reflexivität bewusstmachen, wie wir sie unter zweitens dieses Beitrags als Kennzeichen partizipativer Forschung erläutern?

- Können alle Mitforschenden einer Forschungsgemeinschaft für reflexive Praxen gewonnen werden? Was setzt Reflektieren in einer Forschungsgemeinschaft voraus? Sind es Kenntnisse, Fähigkeiten oder Erfahrungen, die zum Beispiel Fachkräfte aus ihrer beruflichen Praxis mitbringen, aber Mitforschende ohne professionellen Hintergrund überfordern?

- Wie lässt sich kritische Reflexivität in Forschungsgemeinschaften fördern und etablieren? Wie kann ein reflexiver Prozess moderiert und begleitet werden, sodass diese Qualität an Reflexivität entsteht?

Um reflexive Praxen und Formate in der partizipativen Forschung weiterzuentwickeln, regen wir an, auch Kenntnisse benachbarter Disziplinen in Betracht zu ziehen. Partizipativ zu forschen erfordert im Kontakt zu sein und setzt deshalb die Fähigkeit zur Selbstreflexion und ein Verständnis von gruppendynamischen Prozessen und Rollen voraus. Dafür verweisen Bergold und Thomas (2012) darauf, sich an Methoden wie der Themenzentrierten Interaktion (TZI) zu orientieren, die einige Instrumente 
zum Verständnis und zur Reflexion von Gruppenprozessen hervorgebracht hat. Die TZI sowie auch die Supervision, zu deren Kerngeschäft das Reflektieren zählt, sind psychoanalytisch verortet und orientieren sich in der Analyse von sozialen Beziehungen an Modellen der Gruppen- und Psychodynamik (Cohn 2009; König \& Schattenhofer 2015). In der Supervision ist Reflexion grundlegendes Arbeitsprinzip (Hausinger 2010). Die Supervision bedient sich dabei eines sehr ausdifferenzierten Verständnisses von Reflexivität und stellt ein breites Repertoire an Instrumenten für reflexive Prozesse zur Verfügung. Für die partizipative Forschung scheint es hier wichtige Ansatzpunkte zu geben, die die Perspektiven und Horizonte für eine reflexive Praxis zu erweitern vermögen (Jacobs 2008).

\section{Danksagung}

Unser Dank gilt allen Mitgliedern und Partner*innen des Forschungsverbundes PartKommPlus. Ganz besonders möchten wir uns bei den Kolleginnen bedanken, die an dem Workshop zum Thema Reflexion im Rahmen eines Kolloquiums des Forschungsverbundes teilnahmen: Katharina Friederich (Hochschule Fulda) vom Projekt „Age4Health “" ${ }^{\text {" }}$ Alexandra Schüssler und Jan Fischer (Landesvereinigung für Gesundheit und Akademie für Sozialmedizin Niedersachsen e. V.) vom Projekt „PEPBS ${ }^{{ }^{5}}$, Christa Böhme und Thomas Franke (Deutsches Institut für Urbanistik) vom Projekt „ $\mathrm{K}^{3{ }^{36}}$ sowie Jane Springett und Hans Wolter aus dem Beirat des Verbundes. Der Austausch und das Einbringen der jeweiligen projektbezogenen Erfahrungen in diesem Workshop trugen wesentlich zur Entstehung des Beitrags bei. Bedanken wollen wir uns auch beim BMBF, das den Forschungsverbund PartKommPlus, aus dessen Gesamtzusammenhang dieser Beitrag entstand, unter dem Förderkennzeichen 01EL1823A-H fördert.

4 Gesunde Stadtteile für Ältere.

5 Partizipative Evaluation der Präventionskette Braunschweig.

6 Kommunen und Krankenkassen - Kooperationen für gesunde Lebenswelten vor Ort. 


\section{Literatur}

Alice Salomon Hochschule (ASH) \& Gesundheit Berlin Brandenburg (GBB) (2016). Begleitmaterialien zum Peerforschungsprozess ElfE: Eltern fragen Eltern, Merkblatt 3: Die (ungleiche) Entwicklung von Kindern allgemein und in Bezug auf den Kita-Besuch. http:// partkommplus.de/fileadmin/files/Dokumente/ElfE/Merkblaetter-ElfE/1_ElfE_Merkblatt_Studienlage_Entwicklung_von_Kindern_und_Kita-Besuch.pdf [Zugriff: 26. Januar 2020].

Altrichter, Herbert (2008). Komplexe praktische Tätigkeit braucht Forschung. Aktionsforschung und Weiterentwicklung beruflichen Handelns. In Hannes Krall, Erika Mikula \& Wolfgang Jansche (Hrsg.), Supervision und Coaching (S.269-284). Wiesbaden: VS Verlag für Sozialwissenschaften.

Apgar, J. Marina; Allen, Will; Albert, Joelle; Douthwaite, Boru; Paz Ybarnegaray, Rodrigo \& Lunda, Jeston (2017). Getting beneath the surface in program planning, monitoring and evaluation: Learning from use of participatory action research and theory of change in the CGIAR Research Program on Aquatic Agricultural Systems. Action Research, 15(1), 15-34.

Bergold, Jarg \& Thomas, Stefan (2012). Partizipative Forschungsmethoden: Ein methodischer Ansatz in Bewegung. Forum Qualitative Sozialforschung / Forum: Qualitative Social Research, 13(1), Art. 30. doi:10.17169/fqs-13.1.1801.

Bisset, Sherri; Tremblay, Marie-Claude; Wright, Michael T.; Poland, Blake \& Frohlich, Katherine (2015). Can reflexivity be learned? An experience with tobacco control practioners in Canada. Health Promotion International, 32(1), 1-10.

Borg, Marit; Karlsson, Bengt; Kim, Hesook Suzie \& McCormack, Brendan (2012). Opening up for Many Voices in Knowledge Construction. Forum Qualitative Sozialforschung / Forum: Qualitative Social Research, 13(1), Art.1, http://dx.doi.org/10.17169/fqs-13.1.1793 [Zugriff: 06. März 2020].

Chiu, Lai Fong (2006). Critical reflection: More than nuts and bolts. Action Research, 4(2), 183-203.

Cohn, Ruth (2009). Von der Psychoanalyse zur themenzentrierten Interaktion. Von der Behandlung einzelner zu einer Pädagogik für alle. 16., durchgesehene Aufl. Stuttgart: Klett-Cotta.

Cook, Tina; Atkin, Helen \& Wilcockson, Jane (2018). Participatory Research Into Inclusive Practice: Improving Services for People With Long Term Neurological Conditions. Forum Qualitative Sozialforschung / Forum: Qualitative Social Research, 19(1), Art. 4, doi:10.17169/fqs-19.1.2667.

Dorozenko, Kate P.; Bishop, Brian J. \& Roberts, Lynne D. (2016). Fumblings and faux pas: Reflections on attempting to engage in participatory research with people with an intellectual disability. Journal of Intellectual \& Developmental Disability, 41(3), 197-208.

Engage for Equity (2019). The CBPR Model. https://engageforequity.org/ [Zugriff: 26. Januar 2020].

Hausinger, Brigitte (2010). Supervision. In Deutsche Gesellschaft für Supervision e. V. (Hrsg.), Kernkompetenz: Supervision. Ausgewählte Formate der Beratung in der Arbeitswelt (S. 43-48). Köln: DGSv.

Jacobs, Gaby C. (2008). The development of critical being? Reflection and reflexivity in an action learning programme for health promotion practitioners in the Netherlands. Action Learning: Research and Practice, 5(3), 221-235. 
Koch, Tina; Mann, Susan; Kralik, Debbie \& van Loon, Antonia M. (2005). Reflection: Look, think and act cycles in participatory action research. Journal of Research in Nursing, 10(3), 261-278.

König, Oliver \& Schattenhofer, Karl (2015). Einführung in die Gruppendynamik. 7. Aufl. Heidelberg: Carl-Auer.

Kümpers, Susanne; Kühnemund, Christina \& Nemelka, Miguel (2017). Partizipative Gesundheitsförderung für und mit älteren Menschen in der Kommune - Das Forschungsprojekt „Age4Health - Gesunde Stadtteile für Ältere“ In Monika Alisch; Stephanie Hagspihl; Claudia Kreipl \& Martina Ritter (Hrsg.), Alter(n) und Soziale Nachhaltigkeit. Interdisziplinäre Zugänge zu den Herausforderungen alternder Gesellschaften (S. 59-79). Kassel: kassel university press.

Labonte, Ronald; Feather, Joan \& Hills, Marcia (1999). A story/dialogue method for health promotion knowledge development and evaluation. Health Education Research, 14(1), $39-50$.

Ledwith, Margaret \& Springett, Jane (2010). Participatory practice: Community-based action for transformative change. Bristol, Chicago: Bristol University Press.

Moser, Heinz (1995). Grundlagen der Praxisforschung. Freiburg: Lambertus.

PartKommPlus. (2018). PartKommPlus - Forschungsverbund für gesunde Kommunen. Erkenntnisse und Empfehlungen im Überblick. http://partkommplus.de/fileadmin/files/ Tagung-1/06_PartKommPlus_Ergebnisse_und_Empfehlungen_der_1._Foerderphase_-_Stand_18_01_11.pdf [Zugriff: 26. Januar 2020].

Robert Koch-Institut (RKI) (2008). Beiträge zur Gesundheitsberichterstattung des Bundes. Lebensphasenspezifische Gesundheit von Kindern und Jugendlichen in Deutschland. Ergebnisse des Nationalen Kinder-und Jugendgesundheitssurveys (KiGGS). Berlin. https://www.rki.de/DE/Content/Gesundheitsmonitoring/Gesundheitsberichterstattung/ GBEDownloadsB/KiGGS_SVR.pdf?__blob=publicationFile [Zugriff: 26. Januar 2020].

Schaefer, Ina; Bär, Gesine \& die Mitglieder des Forschungsprojektes ElfE (2019). Die Auswertung qualitativer Daten mit Peerforschenden. Ein Anwendungsbeispiel aus der partizipativen Gesundheitsforschung. Forum Qualitative Sozialforschung / Forum: Qualitative Social Research, 20(3). doi:10.17169/FQS-20.3.3350.

Schön, Donald (1983). The reflective practioner. How professionals think in action. London: Maurice Temple Smith Ltd.

Springett, Jane; Wright, Michael T. \& Roche, Brenda (2011). Developing Quality Criteria for Participatory Health Research. WZB Discussion Paper. Berlin: Wissenschaftszentrum Berlin für Sozialforschung.

Tremblay, Marie-Claude; Richard, Lucie; Brousselle, Astrid \& Beaudet, Nicole (2013). Learning reflexivity from a health promotion professional development program in Canada. Health Promotion International, 29(3), 538-548.

von Unger, Hella (2012). Partizipative Gesundheitsforschung: Wer partizipiert woran?. Forum Qualitative Sozialforschung / Forum: Qualitative Social Research, 13(1), Art. 7, http://nbn-resolving.de/urn:nbn:de:0114-fqs120176 [Zugriff: 30. Januar 2020].

Wihofszky, Petra (2013). Die Praxis der Gesundheitsförderung zwischen Top-down und Bottom-up. Ansätze partizipativen Planens und Forschens in der Konzeptentwicklung. Prävention und Gesundheitsförderung, 8(3), 181-190.

Wright, Michael T. (2012). Partizipation in der Praxis: die Herausforderung einer kritisch reflektierten Professionalität. In Rolf Rosenbrock, \& Susanne Hartung (Hrsg.), Handbuch Partizipation und Gesundheit (S.91-101). Bern: Huber. 
Wright, Michael T. (2013). Was ist Partizipative Gesundheitsforschung? Positionspapier der International Collaboration for Participatory Health Research. Prävention und Gesundheitsförderung, 8(3), 122-131.

Wright, Michael T. (2016). Partizipative Gesundheitsforschung. In Bundeszentrale für gesundheitliche Aufklärung (BZgA) (Hrsg.), Leitbegriffe der Gesundheitsförderung. https:// www.leitbegriffe.bzga.de/alphabetisches-verzeichnis/partizipative-gesundheitsforschung/ [Zugriff: 26. Januar 2020].

Wright, Michael T.; Cook, Tina; Springett, Jane \& Kongats, Krystyna (2018a). Building Consensus, Celebrating Diversity: The International Collaboration for Participatory Health Research. In Michael T. Wright \& Krystyna Kongats (Hrsg.), Participatory Health Research.Voices from Around the World (S. 17-24). New York: Springer.

Wright, Michael T.; Hartung, Susanne; Bach, Mario; Brandes, Sven; Gebhardt, Birte; Jordan, Susanne; Schaefer, Ina \& Wihofszky, Petra (2018b). Impact and Lessons Learned from a National Consortium for Participatory Health Research: PartKommPlus - German Research Consortium for Healthy Communities (2015-2018). BioMed Research International 2018, doi:10.1155/2018/5184316.

Open Access Dieses Kapitel wird unter der Creative Commons Namensnennung 4.0 International Lizenz (http://creativecommons.org/licenses/by/4.0/deed.de) veröffentlicht, welche die Nutzung, Vervielfältigung, Bearbeitung, Verbreitung und Wiedergabe in jeglichem Medium und Format erlaubt, sofern Sie den/die ursprünglichen Autor(en) und die Quelle ordnungsgemäß nennen, einen Link zur Creative Commons Lizenz beifügen und angeben, ob Änderungen vorgenommen wurden.

Die in diesem Kapitel enthaltenen Bilder und sonstiges Drittmaterial unterliegen ebenfalls der genannten Creative Commons Lizenz, sofern sich aus der Abbildungslegende nichts anderes ergibt. Sofern das betreffende Material nicht unter der genannten Creative Commons Lizenz steht und die betreffende Handlung nicht nach gesetzlichen Vorschriften erlaubt ist, ist für die oben aufgeführten Weiterverwendungen des Materials die Einwilligung des jeweiligen Rechteinhabers einzuholen.

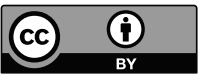

